\title{
Determination of factors affecting abrasion resistance to garments care performance with coated fabrics
}

\author{
[Ada Traumann*, Merje Beilmann¹, Diana Tuulik², Marika-My Laid³]
}

\begin{abstract}
The development of textile industry has been varied during last years. Fabrics are subjected to abrasion during their life cycle using different types of fibres, finishing agents added to fibres and/or fabrics, changing construction or thickness of fabrics or several other factors that can increase the mechanical properties of fabrics. In this study, the properties of brand new filament fabrics have been compared to finished garments` fabrics after different maintenance possibilities. The Martindale tester is used according to standard EN ISO 12947 [1]. For assessment, the specimen is examined at suitable intervals to see whether two threads have broken, while recording the number of cycles. The abrasion resistance is only one of several factors contributing to wear performance or durability of fabrics. The inherent mechanical properties of fibres are important, elastic recovery of fibres or high elongation, hence a high degree of quality. Longer fibres indicate better abrasion resistance than short fibres. Flat plane weave fabrics have better resistance than other weaves because yarns are more tightly locked. However, better mechanical and chemical properties of fabrics can disappear when the wearer does not follow the garment care procedures correctly. Based on this study, it can be concluded that the abrasion resistance of fabrics stays the same after false maintenance like using too high temperatures for washing and drying at home.
\end{abstract}

Keywords - filament fibres fabrics, coated or laminated materials, abrasion resistance, tearing strength, maintenance of textile garments

\section{Introduction}

Nowadays, woven fabrics have a very wide range of applications. Textile industry is in the transition zone between a traditional textile production and the realisation of highly focused design and production of added values textiles [1]. Depending on the application of fabrics, a variety of factors are used, such as the type of fibre, dimensions of fibres, the structure of yarns, type and amount of finishing agents added to fibres, yarns or fabrics. Polyester is a widely known durable fibre. It is also a cheap fibre but this knowledge has changed after the emergence of polyester micro fibres that are more luxurious and expensive.

The aim of this study was to investigate the fabrics of children's outerwear. Polyester fibres and fabrics have many uses since they are strong, easily washed and quick drying and are resistant to most chemicals and abrasion.

Polyester is often used for outerwear because of its high durability. Its hydrophobic property makes it ideal for garments that are used to be in wet or dump environmentscoating fabric with water-resistant or stain-resistant finishes intensifies this effect.
Consumer's launder and maintenance of textile garments has important impact in the lifespan of textiles [2]. The most common is machine washing and dehumidification in the dryer. Because children play outside whether in rain or sunshine, their clothes may need machine wash routinely. Nowadays, kindergartens use drying boxes to get dry clothes fast before going home. But we do not have the information about what kind of conditions of machine wash and dry they use. It was also important to investigate whether domestic care affects properties of outerwear fabrics and their lifespan. Some mechanical and chemical properties of fabrics can disappear when the wearer does not follow the garment care procedures correctly. Usually, the maintenance information is marked on the care label of each garment but sometimes consumers do not pay attention to this or they are aware of the infringement. For example, using higher temperature than allowed in order to get dry clothes faster.

On the basis of above, four different test fabrics were selected and two different domestic maintenances carried out. One of them was according to care label information and for the second, higher temperature of $65^{\circ} \mathrm{C}$ was selected for the drying. The washing temperature was kept according to the information on the care label at $40^{\circ} \mathrm{C}$.

\section{Materials}

Test materials were selected from fall/winter and spring/summer collections, two different from each. Material composition was $100 \%$ polyester (PES) coated with polyurethane (PU). Test materials were plain-weave fabrics and one of them was additionally printed. Data of test materials are presented in Tab. 1.

TABLE 1
\begin{tabular}{|c|c|c|c|c|c|}
\hline Article & $\begin{array}{c}\text { Density } \\
\left(\mathbf{g} / \mathbf{c m}^{\mathbf{2}}\right.\end{array}$ & $\begin{array}{c}\text { Laminate/ } \\
\mathbf{m m}\end{array}$ & $\begin{array}{c}\text { Weave } \\
\text { structure }\end{array}$ & Composition & $\begin{array}{c}\text { Colour } \\
\text { fastness }\end{array}$ \\
\hline PB1215 & 180 & PU/0,22 & $150 \mathrm{D} * 300 \mathrm{D}$ & $100 \% \mathrm{PES}$ & $3 \mathrm{~K}$ \\
\hline PB04 & 95 & $\mathrm{PU}$ & $75 \mathrm{D} * 75 \mathrm{D} * 240 \mathrm{~T}$ & $100 \% \mathrm{PES}$ & $\mathrm{ND} *$ \\
\hline PB04PR & 95 & $\mathrm{PU}$ & $75 \mathrm{D} * 75 \mathrm{D} * 240 \mathrm{~T}$ & $100 \% \mathrm{PES}$ & $\mathrm{ND} *$ \\
\hline $\mathrm{Q} 2366$ & 180 & $\mathrm{PU} / 0,22$ & $150 \mathrm{D} * 300 \mathrm{D}$ & $100 \% \mathrm{PES}$ & $5 \mathrm{~K}$ \\
\hline
\end{tabular}

*ND- no data

The fibre itself is woven into a yarn. There are two types of polyester yarns: filament and spun. The selected fabrics for testing were woven of continuous multi-filament yarns.

Multifilament yarns are made by taking the single polyester filaments, grouping them together and then twisting or air-entangling them to make them applicable. The spun 
Proc. of the Fourth International Conference on Advances in Social Science, Management and Human Behaviour - SMHB 2016. Copyright (C) Institute of Research Engineers and Doctors. All rights reserved.

ISBN: 978-1-63248-116-0 doi: 10.15224/ 978-1-63248-116-0-39

yarns are produced in much the same way as a cotton or wool yarn is produced. The long filaments are first cut into short pieces called staple. These are then combined together and spun to create a yarn made up of thousands of short filaments. [3]

The filament fibres are long and strong, therefore do not get tangled with each other during the friction. Therefore, the surface of the fabric is not hairy. The surface of the test material is homogeneously worn out, but appearance is unharmed and smooth.

Moisture has a big impact on thermal comfort, but also on sensory comfort. This sensory comfort may change with different activity rates and environmental conditions. Moisture from external sources (e.g. rain) will impact both the microand macro-environments, which in turn impacts the comfort perception of both hot and cold conditions.[4]

Fabrics of outerwear are usually developed waterproof, breathable and laminated. The back side of the fabric is coated with a thin microporous layer. These kind fabrics are called poromeric or microporous fabrics. The membranes stretch in both directions, and there are micro-pores small enough to allow the vapour but not the water flow through the pores. The simplest form is a two-layered fabric in which the outer layer is typically composed of polyester. The inner membrane layer is made of polyurethane.

Two different coating types from our fabrics have been used based on the manufacturer's information. One of them is called Milky laminate, for which the coating paste is placed on the back of the fabric and after that, it is scrubbed into the fabric. The other method, the most common one, is lamination in which the prepared polymer layer is attached to the fabric by using an adhesive or by heating lightly the inner side of the polymer before the layers are joined. The main deficiency is that the microporous fabrics are then soiled, after that the micro-pores may open up and are filled with dirt.

\section{Methods}

Since the test materials are used for the production of children's wear, it was important to carry out tests on fabric abrasion resistance. Additionally, a tearing test of the same materials was conducted using the Elmendorf method. Kids are good for testing garment wear but this time, the emphasis was on laboratory tests of basic fabrics.

The aim of this study was to test the outer fabric in the conditions of home care and thereafter to carry out tests on the abrasion resistance and the tearing of cared fabrics. One washing and two different drying conditions were selected to simulate a home care regime, see in Tab.2. The quantity of the gel detergent in millilitres $(\mathrm{ml})$ was chosen according to medium water hardness/ softness 4-14 dH. The recommended amount of gel detergent for the average dirty laundry was taken from the manufacturer's data sheet, see in Tab.2. The washing gel does not contain odorants or colorants. The chemical composition is suitable for washing the laundry of small children and allergy-prone persons [5]. Wash tests were carried out in the domestic washing machine with the custom wash program for sportswear and the spin speed to remove the water from the fabric`s tests at the end of a cycle was $800 \mathrm{rpm}$ (revolutions per minutes).

TABLE 2 WASHING AND DRYING CONDITIONS FOR TEST MATERIALS

\begin{tabular}{|c|c|c|c|c|c|}
\hline \multirow{2}{*}{ Maintenance } & \multirow{2}{*}{$\begin{array}{c}\text { Detergent, } \\
\text { ml }\end{array}$} & \multicolumn{2}{|c|}{$\begin{array}{c}\text { Conditions of } \\
\text { washing }\end{array}$} & \multicolumn{2}{|c|}{$\begin{array}{c}\text { Conditions of } \\
\text { drying }\end{array}$} \\
\cline { 3 - 6 } & & Temp, $\boldsymbol{t}^{\boldsymbol{o}}$ & $\begin{array}{c}\text { Term, } \\
\text { in min }\end{array}$ & Place & Temp, $\boldsymbol{t}^{\boldsymbol{o}}$ \\
\hline Correct (C) & 75 & 40 & 44 & In the air & $20-22$ \\
\hline Incorrect (IC) & 75 & 40 & 44 & $\begin{array}{c}\text { In the } \\
\text { drying } \\
\text { box }\end{array}$ & 60 \\
\hline
\end{tabular}

The correct maintenance was carried out according to the care label instructions of the fabric manufacturer, see in Tab. 2. During the incorrect maintenance, the temperature of the dryer was changed to higher than allowed, to $60^{\circ} \mathrm{C}$.

From each of the four fabric articles, test specimens were collected, where the first test specimen was taken from the brand-new fabric. The second and the third test specimens were collected in accordance with the maintenance mode, correct and incorrect. The home maintenance cycle was carried out five times before the testing.

\section{A. Standard atmospheres for testing}

An air-conditioned laboratory shall provide and maintain a standard atmosphere. Standard atmosphere shall have a temperature of $20{ }^{\circ} \mathrm{C}$ and relative humidity of $65.0 \%$. The tolerance for temperature is allowed $\pm 2.0{ }^{\circ} \mathrm{C}$ and $\pm 4.0 \%$ for relative humidity.

Variations in temperature and relative humidity are likely to exist throughout the working space. Our laboratory indoor climatic conditions were at the time of the testing the following: the temperature was $25^{\circ} \mathrm{C}$ and relative humidity $32 \%$.

The specific condition of temperature and relative humidity are considered attained when the following requirements have been satisfied. The mean temperature and relative humidity over any continuous $1 \mathrm{~h}$ period shall conform to the tolerance zone at the standard atmosphere conditions. The spatial variation of the standard atmosphere shall comply with the specified tolerance zone.[6]

\section{B. Determination of the abrasion resistance of fabrics}

It is possible to choose two different types of Martindale method according to ISO 12947:1998. Part 2 is Determination of specimen breakdown and Part 3 is Determination of mass loss. The third possibility is visual examination of textiles using ordinal characteristics according ISO 5725. Tests of this study were carried out according to part 2, where the evaluation of the abrasion resistance of woven fabric is determined from the inspection interval to breakdown of the specimens. The Martindale abrasion tester subjects a circular specimen to a defined load and rubs it against an abrasive medium (i.e standard fabric) in a translational movement 
Proc. of the Fourth International Conference on Advances in Social Science, Management and Human Behaviour - SMHB 2016. Copyright $(\odot$ Institute of Research Engineers and Doctors. All rights reserved. ISBN: 978-1-63248-116-0 doi: 10.15224/ 978-1-63248-116-0-39

tracing a Lissajous figure. The specimen is subjected to abrasive wear for a predetermined number of rubs.[7]

The test apparatus, a 4-head Martindale abrasion tester, consists of a baseplate on which the abrading tables and a drive mechanism are mounted, see on Fig. 1. The abradant was woven wool mounted over felt. The small test specimen is placed on the large abradant and then cycled backwards and forwards in a Lissajous motion producing even wear. To the top of specimen a force of $9 \mathrm{kPa}$ was applied to hold it against the abradant.

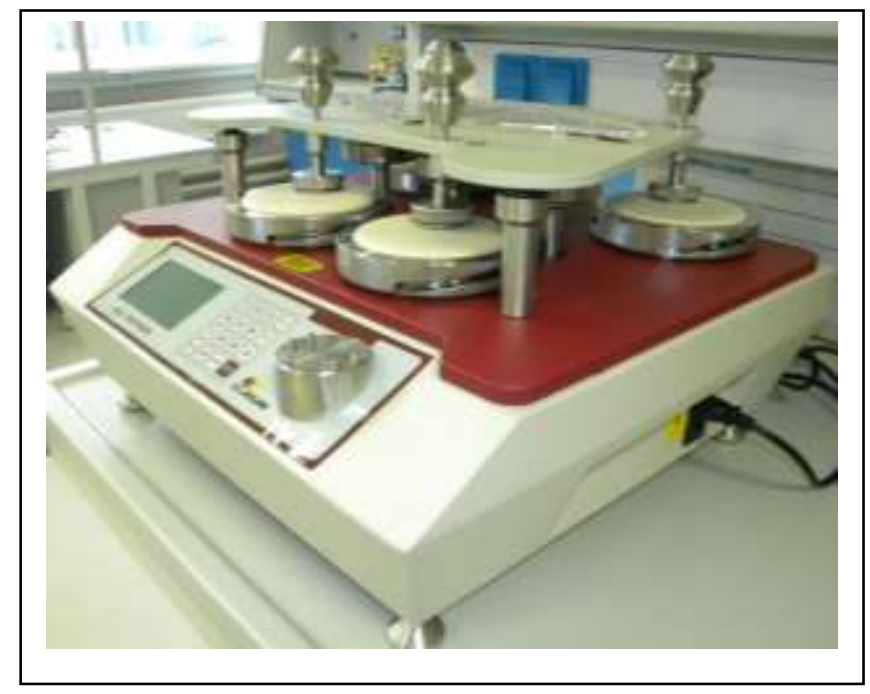

Figure 1. 4 Head Martindale Abrasion Tester

For the test fabrics of this study, the number of rubs according to the relevant test series given in Tab. 3 have been selected. For diagnostic purposes, the test interval for each test series may be reduced as the end point is approached.

TABLE 3 TEST INTERVALS FOR ABRASION TESTING

\begin{tabular}{|c|c|c|}
\hline Test series & Number of rubs & Test interval (rubs) \\
\hline a & 5000 & 0 \\
\hline b & 25000 & 0 \\
\hline c & 50000 & after 50000 \\
\hline d & 100000 & to the end \\
\hline
\end{tabular}

Different fabric structures will require different inspection intervals. Alternative test intervals have been chosen as you can see in Tab.3, which was agreed upon by the interested parties of this study. After 50000 rubs, the abradant was replaced.

\section{Determination of tear strength}

The measurements of tear strength were carried out according to the international standard EN ISO 13937:2000. This standard specifies the methods for the determination of tear force of fabrics. Part 1 describes a ballistic pendulum method (Elmendorf) for the determination of tear force of textile fabrics. The method describes the measurement of the tear force required to propagate a single-rip tear of defined length from a cut in a fabric when a sudden force is applied. Tests are mainly applicable to woven textile fabrics.[8]

The falling (ballistic) pendulum (Elmendorf) method is used for the determination of the average force required to single-rip-tear of a fabric specimen by the force of a falling pendulum. Each test specimen has a notch in the long side. For test specimens, where the short side is parallel to the warp, the direction of the tear is qualified as across weft and where the short side is parallel to the weft, the tear is qualified as across warp [8].

The ballistic pendulum measures energy directly. In current practice, it is preferred to express tear resistance as a force which is usually indicated directly in newton $(\mathrm{N})$. To describe the results, the arithmetic mean of the tear force is calculated in newton and at least five specimens tested for each directions.[8]

\section{Discussion of Results}

A fabric's resistance to abrasion and tear strength are affected by many factors, such as fibre type, the inherent mechanical properties of the fibres, the structure of yarns, the construction and thickness of the fabrics, and the type and amount of finishing material added to the fibres, yarns or fabrics.[9]

\section{A. Fabric abrasion resistance}

Analysing the results of fabric abrasion resistance, it was concluded that for each test series, the end point/breakdown was not approached. Also the pilling could not be observed from the specimens.

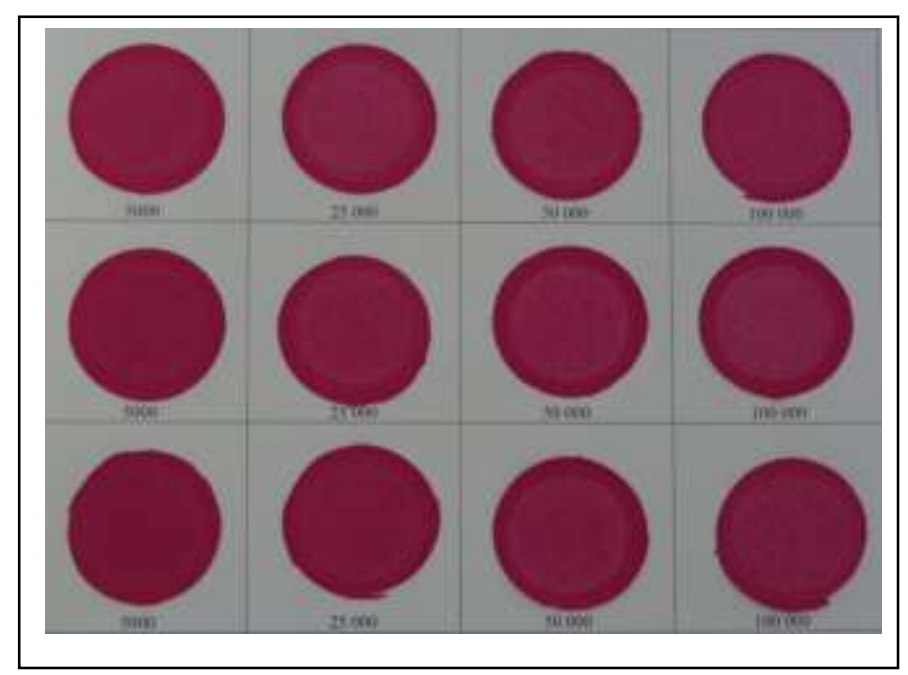

Figure 3. Determination of fabric resistance art. PB1215

For each fabric article, tests of abrasion resistance were provided by the same system as you can see on Fig. 3. In the first row, there are test specimens of a brand-new (BN) fabric. In the second row, there are test specimens of correct (C) maintenance and in the third row, of incorrect (IC) maintenance (see in Tab.2). Outcomes are visually the same 
Proc. of the Fourth International Conference on Advances in Social Science, Management and Human Behaviour - SMHB 2016. Copyright $(\odot$ Institute of Research Engineers and Doctors. All rights reserved.

ISBN: 978-1-63248-116-0 doi: 10.15224/ 978-1-63248-116-0-39

and the abrasion resistance results do not differ for cared and brand-new test specimens. Only a change in shade can be detected. If you compare three types of test specimens whose number of rubs is finally 100000 rubs then they are not different as you can see on Fig 4. A digital microscope MiScope with the magnification of 130 times was used on Fig.4.

Similar visual results, as you can see on Fig. 3 were observed for all three fabric articles.

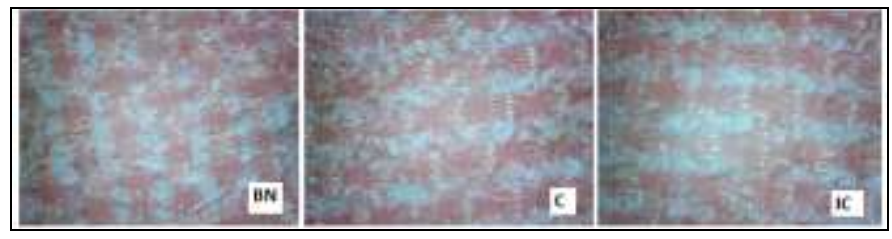

Figure 4. Three different test specimens (BN-brand new, C-correct, ICincorrect) of art. PB04 on rubs 100000

Our test material composition was $100 \%$ polyester (PES) and plain-weave fabrics.

Based on the earlier scientific results, it can be concluded that polyester is generally considered to have auspicious abrasion resistance. Longer filament fibres incorporated into a fabric show better abrasion resistance than short fibres because it is harder to extract them from the fabric structure. Flat plane-weave fabrics are more tightly locked in a plain weave structure. [9]

As one of four fabrics, article PB04PR, was printed, the print quality of the three test specimens is rather abraded on rubs 100 000, see on Fig.5.

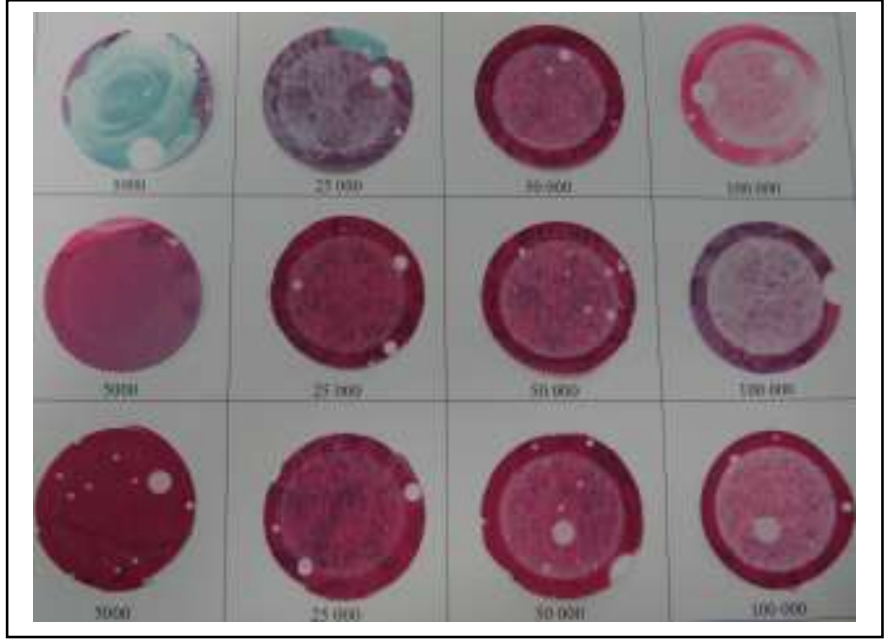

Figure 5. Test specimens of art. PB04PR in different rubs.

\section{B. Fabric tear strength}

For the measurements of tear strength, the appropriate measurement methods were chosen by taking into account the fabric's destination, the kind of tear performance and also the parameters and criteria which are in the form of normative documents.
Previous tests have shown that filament yarn has higher tear strength compared to staple yarn. An increase in yarn density in woven fabrics will decrease the tear strength of a fabric. [9]

For representing the results, the arithmetic mean of the tear force in newton $(\mathrm{N})$ has been calculated, see in Tab.4.

TABLE 4 TEAR STRENGHT RESULTS
\begin{tabular}{|c|c|c|c|c|c|c|}
\hline \multirow{2}{*}{ Article } & \multicolumn{2}{|c|}{$\begin{array}{c}\text { Brand-new ( BN) } \\
\text { fabric, N }\end{array}$} & \multicolumn{2}{c|}{$\begin{array}{c}\text { Correct (C) } \\
\text { maintenance, N }\end{array}$} & \multicolumn{2}{c|}{$\begin{array}{c}\text { Incorrect (IC) } \\
\text { maintenance, N }\end{array}$} \\
\cline { 2 - 7 } & Weft & Warp & Weft & Warp & Weft & Warp \\
\hline PB1215 & 36.01 & 16.95 & 40.75 & 18.20 & 39.41 & 18.27 \\
\hline PB04 & 6.34 & 8.41 & 6.10 & 8.31 & 5.99 & 8.12 \\
\hline PB04PR & 6.86 & 5.37 & 6.04 & 6.89 & 5.56 & 6.94 \\
\hline Q2366 & 27.32 & 16.31 & 30.33 & 17.44 & 30.04 & 17.08 \\
\hline
\end{tabular}

On the following Figures 7-9 results of tear strength for weft and warp per different test specimens has been given.

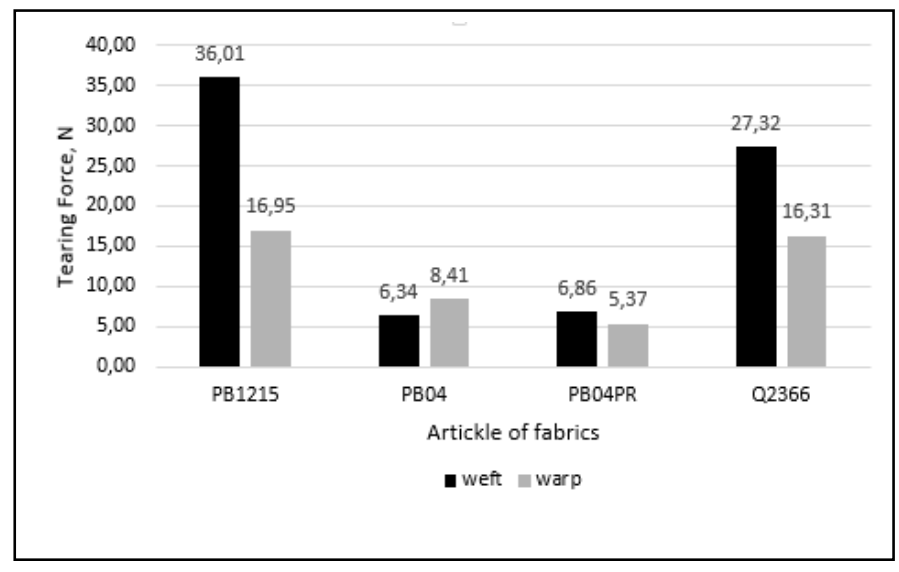

Figure 7. Tear strength results of brand-new fabrics

The relationships between the mean tear strength and the density of fabric are direct according to Fig.7- 9. For thicker fabrics like art. PB1215 and art. Q2366 at the density $180 \mathrm{~g} / \mathrm{m}^{2}$ is needed to implement the bigger tearing force.

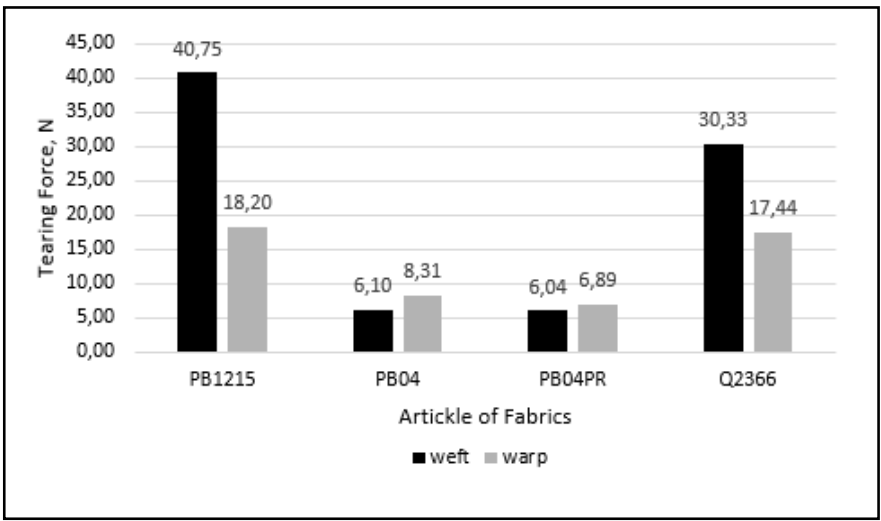

Figure 8. Tear strength results of fabrics correct maintenance 
Proc. of the Fourth International Conference on Advances in Social Science, Management and Human Behaviour - SMHB 2016. Copyright (C) Institute of Research Engineers and Doctors. All rights reserved. ISBN: 978-1-63248-116-0 doi: 10.15224/978-1-63248-116-0-39

The maintenance of thicker fabrics do not degrade the tear strength, but will make it even better, up to $10 \%$, as we can see on Fig. 8 -9. There is no difference in tearing strength between correct and incorrect maintenance for all four test fabrics.

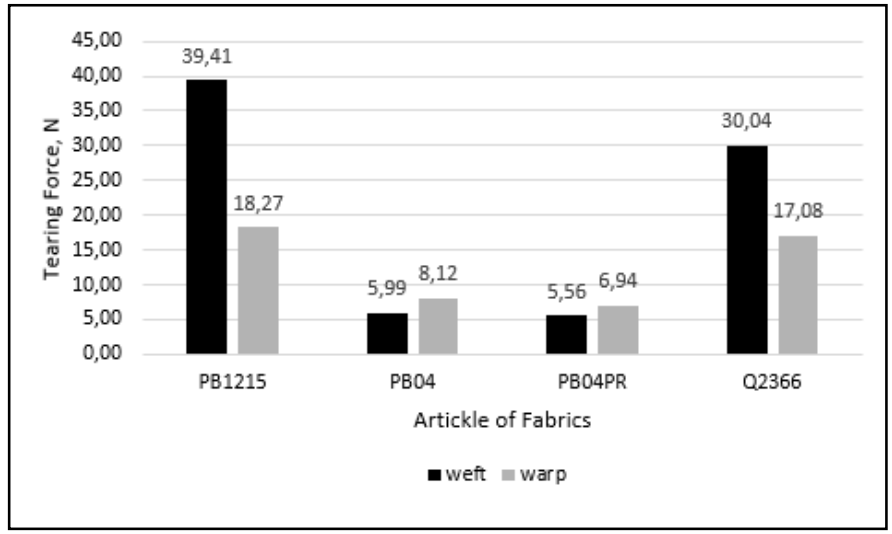

Figure 9. Tear strength results of fabrics incorrect maintenance

Nevertheless, upon analysing the results for four fabrics, we cannot state unequivocally whether such an interpretation would be useful for a bigger sample population, nor whether any kind of 'anomaly' (e.g. for the same fabric, the result for the warp direction can be once higher and once lower than for the weft direction) is due to the yarn strength uniformity, or whether it is a regularity, which can occur for some fabrics of given technological parameters.[10]

\section{v. Conclusions}

Based on the results, the following conclusions can be made:

- abrasion resistance does not degrade after five home care procedures

- there is a linear correlation between the density of fabrics and tear strength

- the maintenance does not degrade the tear strength of fabrics

On the basis of the results above, it can be concluded that the domestic care of children's outerwear fabrics does not make the mechanical properties, like abrasion resistance and tear strength, worse. There is even no difference between incorrect and correct maintenance. It is important to investigate next properties of fabrics like tensile strength, water resistance and breathability.

It is also necessary to further test fabrics under the conditions of home care. It is possible that children's clothes that are really dirty, are washed at much higher temperatures and, in order to get clothes dry in the shortest possible time, elevated temperatures are used in a dryer as well.
Ada Traumann*

TTK Tallinn University of Applied Sciences, Faculty of Clothing and Textile Estonia

Merje Beilmann ${ }^{1}$

TTK Tallinn University of Applied Sciences, Faculty of Clothing and Textile Estonia

Diana Tuulik ${ }^{2}$

TTK Tallinn University of Applied Sciences, Faculty of Clothing and Textile Estonia

Marika-My Laid ${ }^{3}$

University of Tartu, Faculty of Economics and Business

Estonia

\section{Acknowledgment}

"The authors would like to thank the company Huppa Ltd for providing material support to this project".

\section{References}

[1] J. HU, K.M. Babu, "Testing intelligent textiles", Fabric testing, Woodhead Publishing in Textiles No 76, 2008, pp.275-827

[2] C. Hurren, "Dyeing and colouring tests for fabrics", Fabric testing, Woodhead Publishing in Textiles No 76, 2008, pp.255-274

[3] Swicofil, "Polyester (PET)", http://www.swicofil.com/pes.html

[4] P.Bishop, "Testing for fabric comfort", Fabric testing, Woodhead Publishing in Textiles No 76, 2008, pp.228-254

[5] Mayeri, Washing Liquid Mayeri Sensitive Color, http://mayeri.eu/en/tooted/household-chemistry/laundrydetergents/washing-liquid-mayeri-sensitive-color/

[6] EVS-EN ISO 139:2005 Textiles- Standard atmospheres for conditioning and testing

[7] ISO 12497:1998 Textiles- Determination of the abrasion resistance of fabrics by the Martindale method

[8] EN ISO 13937:2000 Textiles - Tear properties of fabrics - Part 1: Determination of tear force using ballistic pendulum method (Elmendorf)

[9] X.Wang, X.Liu, C.Hurren, "Physical and mechanical testing of textiles", Fabric testing, Woodhead Publishing in Textiles No 76, 2008, pp.90-124

[10] B. Witkowska, I. Frydrych, "A Comparative Analysis of Tear Strength Methods", FIBRES \& TEXTILES in Eastern Europe, 2004, Vol. 12, No. 2 (46) 